\title{
Learning to play golf for elderly people with subjective memory complaints: feasibility of a single-blinded randomized pilot trial
}

Julia K. Stroehlein ${ }^{1}$, Solveig Vieluf ${ }^{1}$, Philipp Zimmer ${ }^{2}$, Alexander Schenk², Max Oberste ${ }^{3}$, Christian Goelz ${ }^{1}$ Franziska van den Bongard ${ }^{1}$ and Claus Reinsberger ${ }^{1 *}$

\begin{abstract}
Background: Subjective Memory Complaints (SMC) in elderly people due to preclinical Alzheimer's Disease may be associated with dysregulation of the Kynurenine Pathway (KP), with an increase in neurotoxic metabolites that affect cognition. Golf is a challenging sport with high demands on motor, sensory, and cognitive abilities, which might bear the potential to attenuate the pathological changes of preclinical AD. This trial investigated the feasibility of learning to play golf for elderly with cognitive problems and its effects on cognitive functions and the KP.

Methods: In a 22-week single-blinded randomized controlled trial, elderly people with SMC were allocated to the golf ( $n=25,180$ min training/week) or control group $(n=21)$. Primary outcomes were feasibility (golf exam, adherence, adverse events) and general cognitive function (Alzheimer's Disease Assessment Scale). Secondary outcomes include specific cognitive functions (Response Inhibition, Corsi Block Tapping Test, Trail Making Test), KP metabolites and physical performance (6-Minute-Walk-Test). Baseline-adjusted Analysis-of-Covariance was conducted for each outcome.

Results: 42 participants were analyzed. All participants that underwent the golf exam after the intervention passed it (20/23). Attendance rate of the golf intervention was $75 \%$. No adverse events or drop-outs related to the intervention occurred. A significant time*group interaction ( $p=0.012, F=7.050$, Cohen's $d=0.89)$ was found for correct responses on the Response Inhibition task, but not for ADAS-Cog. Moreover, a significant time*group interaction for Quinolinic acid to Tryptophan ratios ( $p=0.022, F=5.769$, Cohen's $d=0.84)$ in favor of the golf group was observed. An uncorrected negative correlation between attendance rate and delta Quinolinic acid to Kynurenic acid ratios in the golf group ( $p=0.039, r=-0.443)$ was found as well.

(Continued on next page)
\end{abstract}

\footnotetext{
* Correspondence: reinsberger@sportmed.uni-paderborn.de

'Department of Sports and Health, Institute of Sports Medicine, Paderborn University, Warburger Straße 100, 33098 Paderborn, Germany

Full list of author information is available at the end of the article
}

(c) The Author(s). 2021 Open Access This article is licensed under a Creative Commons Attribution 4.0 International License, which permits use, sharing, adaptation, distribution and reproduction in any medium or format, as long as you give appropriate credit to the original author(s) and the source, provide a link to the Creative Commons licence, and indicate if changes were made. The images or other third party material in this article are included in the article's Creative Commons licence, unless indicated otherwise in a credit line to the material. If material is not included in the article's Creative Commons licence and your intended use is not permitted by statutory regulation or exceeds the permitted use, you will need to obtain permission directly from the copyright holder. To view a copy of this licence, visit http://creativecommons.org/licenses/by/4.0/. The Creative Commons Public Domain Dedication waiver (http://creativecommons.org/publicdomain/zero/1.0/) applies to the data made available in this article, unless otherwise stated in a credit line to the data. 
(Continued from previous page)

Conclusions: The findings indicate that learning golf is feasible and safe for elderly people with cognitive problems. Preliminary results suggest positive effects on attention and the KP. To explore the whole potential of golfing and its effect on cognitive decline, a larger cohort should be studied over a longer period with higher cardiovascular demands.

Trial registration: The trial was retrospectively registered (2nd July 2018) at the German Clinical Trials Register (DRKS00014921).

Keywords: Golf, Subjective memory complaints, Alzheimer's Disease, Cognitive Performance, Kynurenine pathway

\section{Introduction}

Subjective Memory Complaints (SMC) are common in the elderly population and describe self-reported difficulties with cognitive functions, while objective performance remains normal $[1,2]$. The heterogeneous etiology of SMC impedes a consistent characterization [3], but two distinct groups were broadly described: One, in which SMC are caused by other factors, including psychiatric diseases [4, 5], psychological distress [6] and chronic diseases [7] and the other, in which SMC represent preclinical Alzheimer's Disease (AD) $[2,8,9]$. In the latter group, SMC were associated with neuropathological changes, including amyloid- $\beta$ deposition $[10,11]$ and diminished grey matter volumes in brain regions affected by AD [12]. A meta-analysis also reported an increased risk of developing dementia in elderly people experiencing SMC compared to those without symptoms [2]. Accordingly, SMC might be a risk factor for subsequent development of $\mathrm{AD}$, thus making it a very interesting group to study with respect to prevention of AD.

There is some evidence that the Tryptophan (TRP) consuming Kynurenine Pathway (KP) is dysregulated in $\mathrm{AD}$ and its preclinical manifestation mild cognitive impairment $[13,14]$.

The isoenzymes Indoleamine-2,3-Dioxygenase 1 (IDO1) and Tryptophan 2,3-Dioxygenase (TDO) catalyze the first and rate limiting step of the essential amino acid TRP to Kynurenine (KYN) [13]. While TDO is mainly expressed in the liver, IDO1 can be expressed in various tissues upon stimulation with inflammatory cytokines, such as Interferon- $\gamma$ and Interleukin-6 [15]. The result is an increase of $\mathrm{KYN}$, which is further metabolized to Kynurenic acid (KYNA) or Quinolinic acid (QUINA) [13]. The latter stimulates N-methyl-D-aspartate (NMDA) receptors in the central nervous system with the potential to induce neuronal excitotoxicity, which is one of the key pathological mechanisms of AD [16]. A simplified visualization of the KP is presented in Fig. 1.

The central dysregulation of the KP also seems to manifest in the blood stream, since accumulated serum QUINA levels [17] and serum Kynurenine to
Tryptophan (KYN/TRP) ratios [14] (a surrogate marker of IDO1 activity) were observed in AD patients and women with high cortical amyloid- $\beta$ burden $[11,18]$. In contrast, KYNA rather has a neuroprotective effect in the central nervous system by inhibiting NMDA neurotransmission [16]. Interestingly, reduced plasma KYNA levels were found in AD patients [19].

The dysregulation of the KP and its neurotoxic/neuroprotective downstream metabolites might also affect cognitive functions. In a large cross-sectional study, increased serum KYN/TRP ratios were associated with poor memory performance [20] in elderly people. In another study, higher serum QUINA levels were correlated with worse executive functions in elderly at risk of dementia [21].

Regular exercise has been found to reduce the risk of $\mathrm{AD}$, maintain cognitive performance, and positively influence the KP in healthy populations [22-24]. Metabolically demanding exercise (such as aerobic and strength training) was investigated most frequently in this field. It has the potential to reduce systemic inflammation [25], which results in decreased activity of enzymes regulating the KP [26].

Interestingly, evidence from animal studies suggest a superior effect of exercise and sensory enrichment (known as enriched environment) on neuroplasticity compared to exercise alone [27, 28]. The idea is supported by recent studies in humans, which conducted multicomponent [29] or dancing interventions [30, 31]. In accordance, a recent systematic review suggested that the metabolic demands of exercise are not the sole factor to improve cognitive performance in elderly people [32]. Golf might have similarities to an enriched environment as well, because it combines physical, sensory, cognitive, and social components [33, 34]. Golf provides a low to moderate intensity profile, although walking a golf course contains periods with higher and lower intensities [33]. Besides cardiovascular activity, learning the golf swing requires high demands on hand-eye coordination, static postural as well as sensorimotor control [34, 35]. During a game, strategic planning, maintaining and manipulating information, as well as adapting to changing environmental conditions illustrate 


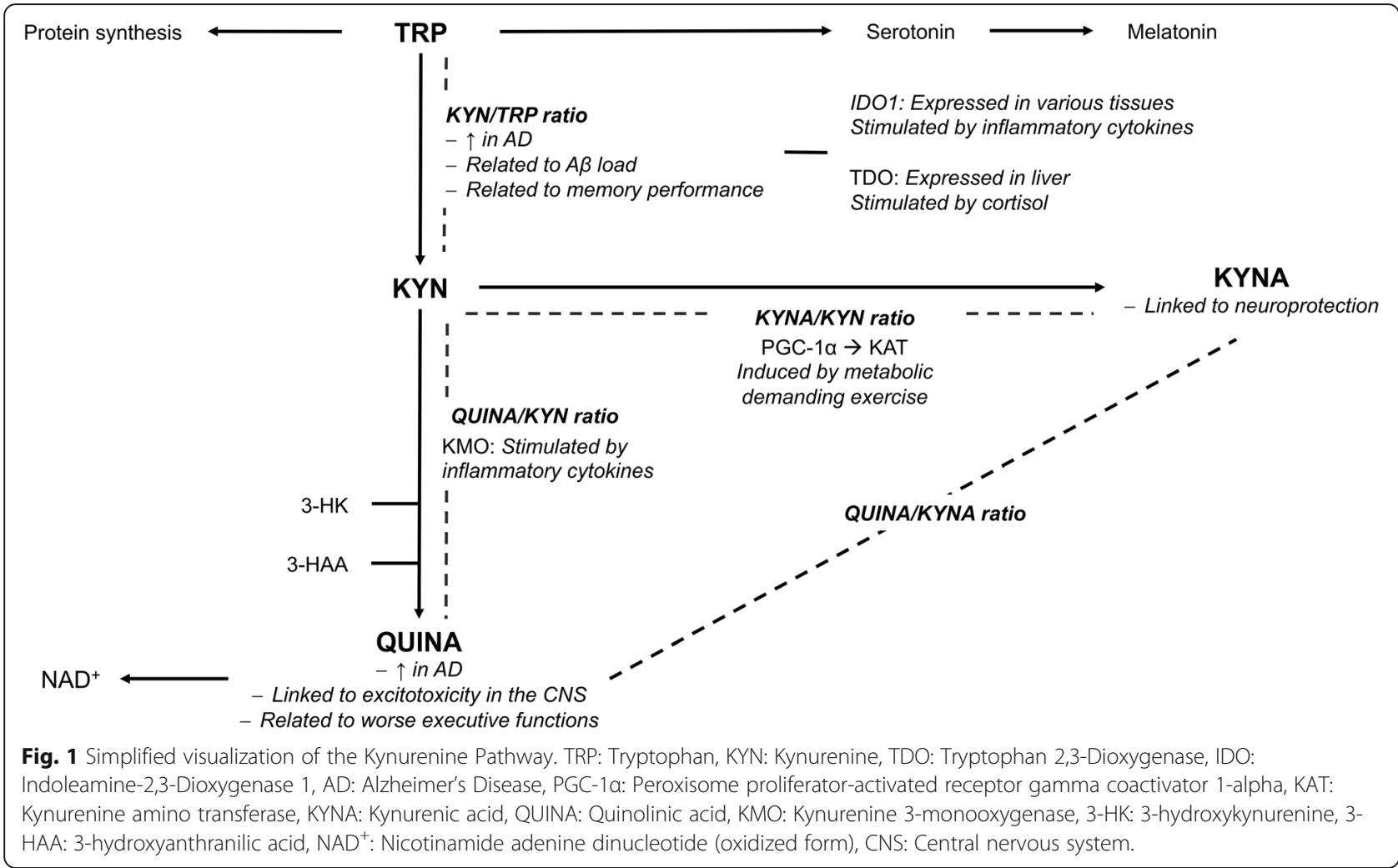

the cognitive demands of golf, including working memory, attention [34] as well as cognitive flexibility. In the social domain, regular golfing facilitates the establishment of social connections and relationships [33]. In sum, the multidimensional profile of learning to play golf might have the potential to positively affect SMCrelated cognitive decline and biological changes in elderly people. However, because of the challenging profile, it remains unclear if elderly people with cognitive problems are able to learn the sports.

Few studies investigated the effects of learning golf on cognitive functions in elderly people. Shimada et al. (2018) [34] reported improvements in immediate and delayed logical memory after a 24-weeks golf intervention with healthy older adults from Japan. Another research group found an increased visual imaginary ability after 20 sessions of golf training in patients with stroke [36].

This study aimed to examine the feasibility of learning to play golf over 22 weeks and its effects on cognitive functions and the regulation of peripheral KP metabolites in elderly people with SMC with no experience in playing golf.

\section{Materials and methods}

The study is reported in accordance with the Consolidated Standards of Reporting Trials (CONSORT) recommendations of 2010 for pilot and feasibility trials [37].

It was designed as a 22-week randomized controlled trial and conducted between May and December 2018 at the Institute of Sports Medicine at Paderborn University. The protocol was approved by the ethics committee of the "Westfälische Wilhelms-Universität Münster". Written informed consent to participate in the study was obtained by each participant before enrollment and was in accordance with the Declaration of Helsinki. Participants were not provided any payment, but the golf group received complimentary golf training.

The trial was retrospectively registered $(02 / 07 / 2018)$ at the German Clinical Trials Register (DRKS00014921).

\section{Screening and eligibility}

Participants were recruited locally via newspapers, social media advertisements and at organizations providing leisure activities for seniors. Exclusion criteria were (1) younger than 60 years, (2) answer "no" to the question: "Do you have subjective memory complaints?" (3) significant experiences in golf (i.e., proficiency certificate or handicap) (4) diagnosed neurological or mental disease or other impairment of physical abilities.

\section{Procedure and randomization}

After inclusion, participants were individually scheduled for baseline data collection before randomization on two 
different days. At day one, the acquisition of medical history and sociodemographic data as well as the neuropsychological testing took place. On the second day, participants underwent a blood sample collection and an endurance performance test. To account for differences in physical activity habits between groups' participants were asked to fill in a questionnaire (Physical Activity Scale for Elderly, PASE, [59]) for two different weeks before randomization and during the 22-week intervention.

After baseline assessments, participants were matched according to age, gender, PASE-score, and Alzheimer's Disease Assessment Scale - Cognitive Subscale (ADAS$\operatorname{Cog}$ ) score [38]. For the matching procedure, the method of randomly permuted blocks was applied to allocate participants with similar characteristics to the golf or control group (1:1 ratio). If a block consisted of one participant or if the number of participants in one block was uneven, the participant was allocated to the golf group. A researcher who was not involved in the neuropsychological tests, blood collection, or golf intervention $(\mathrm{FvdB})$ carried out the random sequence generation and group allocation.

A CONSORT flow chart of the study is presented in Fig. 2.

\section{Subjective memory complaints}

According to current guidelines [39] a self-designed questionnaire without further formal cognitive testing was used to characterize people with SMC. It included the items memory complaints in daily life (yes/no), serious worries about memory complaints (yes/no) and the onset of the complaints (years). The presence of depressive symptoms and anxiety was evaluated with the Beck's Depression Inventory (BDI) [40].

\section{Golf intervention}

The golf training was performed at Paderborn University Golf Club. The golf training sessions were planned,

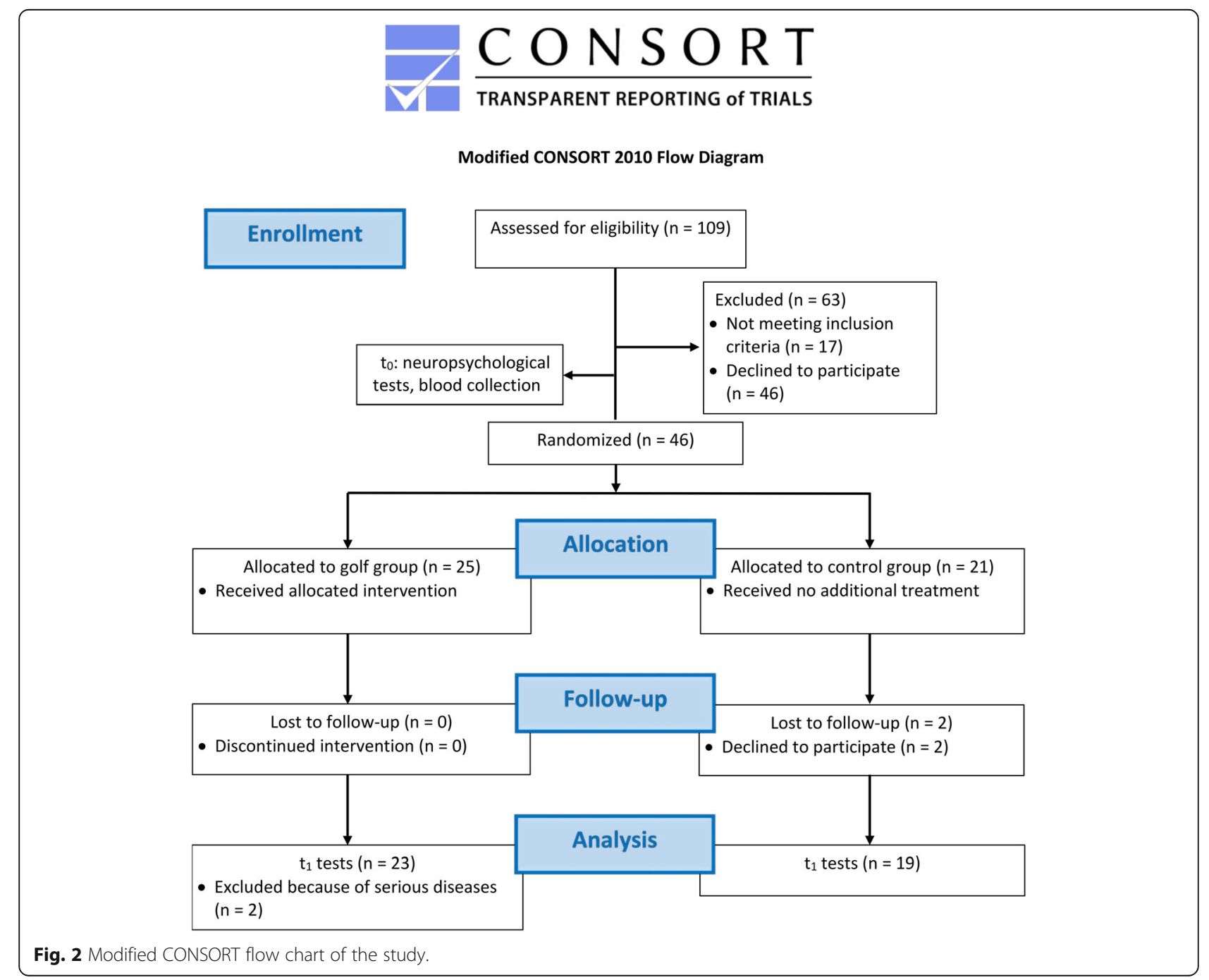


supervised, and mostly conducted by a fully qualified professional golf trainer of the Professional Golfer Association (PGA). In addition, three other golf trainers aided and provided the training sessions. A maximum of 13 participants who were instructed by two trainers was allowed per session. The golf training consisted of three sessions per week, each lasting $60 \mathrm{~min}$ over a period of 22 weeks. Two of three sessions were supervised and instructed by trainers. The third session was not supervised, but participants were asked to practice the previously acquired skills independently at the driving range.

The supervised golf program included 18 practice sessions and 25 sessions at the driving range. All sessions started with a short warm-up (10 min), which consisted of coordination and stretching exercises. In the practice sessions (week 1 to 8), participants learned basic golf techniques, starting with putting and chipping. After 5 weeks, pitching was introduced and practiced and after 7 weeks, participants learned the full golf swing. Golf trainers gave individual feedback to improve the techniques, e.g. via videos or verbal instructions during the sessions. At week 9, participants started to practice at the driving range. All trainers encouraged the participants to practice the golf techniques at home in front of a mirror, to learn the golf rules and to interact with the other participants.

\section{Control group}

The control group was asked to keep their lifestyle and sports activities unchanged for the course of the study. Each control subject was asked to fill out PASE questionnaires every week to monitor any changes.

\section{Primary outcomes: Feasibility and general cognitive performance}

To elucidate if learning to play golf is feasible for elderly people with cognitive problems, the golf group underwent a graded technical exam only at the end of the intervention. This exam is equivalent to the German license to play golf. It included the assessment of the golf techniques putting, chipping, pitching, and the full golf swing performed by the professional golf trainer. A score of 20 points could be achieved for each technique, resulting in a total score of 80 points. Therefore, a higher score was related to better golf performance. Attendance rate was measured by the average number of sessions which were conducted by all participants of the golf group compared to the absolute number of conducted trainings. Safety was measured by adverse events and drop-outs related to the golf intervention.

The neuropsychological tests were conducted at baseline $\left(t_{0}\right)$ and after the 22-week intervention $\left(t_{1}\right)$ by trained study staff not involved in the intervention and blinded to the group assignment. Blinding was ensured by asking participants not to disclose their group assignment to the outcome assessors. General cognitive functions were assessed with the ADAS-Cog [38], which was sensitive to the effects of physical activity in elderly people with SMC in another study [41].

\section{Secondary outcomes: Specific cognitive functions, physical evaluation and the KP}

In addition, three different tests of specific cognitive functions were conducted and consisted of visual-spatial working memory (assessed with Corsi Block Tapping Task), and executive functions (assessed with INHIB Response Inhibition and the Trail Making Test part B, respectively). Except for the ADAS-Cog, all tests were part of the automated computer-based Vienna Test System Version 6.82.000 (Schuhfried GmbH, Mödling, Austria).

To assess endurance performance, the 6-MinuteWalk-Test (6MWT) was conducted [42].

Blood samples via venipuncture were obtained during early and late morning (8-12 am) to investigate the KP and IL-6. Participants were asked to avoid physically demanding activities on the same morning before the blood sample collection. The serum samples were left to clot at room temperature for $30 \mathrm{~min}$. After that, samples were centrifuged for $10 \mathrm{~min}$ at $1800 \mathrm{~g}$ and frozen at $-31{ }^{\circ} \mathrm{C}$ until transportation (max. 2 weeks later).

Blood samples were processed and analyzed as described in detail in Joisten et al. 2020 [43]. High performance liquid chromatography (HPLC) and tandem mass spectrometry (MS/MS) were used to analyze serum concentrations of KP metabolites (KYN, TRP, QUINA, KYNA). Ratios of KYN to TRP, QUINA to KYNA, QUINA to TRP and QUINA to KYN were calculated to indicate changes in the degradation steps of the KP.

IL-6 was measured using the Quantikine high sensitive IL-6 Enzyme-linked Immunosorbent Assay (ELISA, R\&D Systems, Minneapolis, USA) according to the manufacturers protocol.

\section{Statistical analysis}

The sample size calculation was conducted with G*Power [44] and was based on the randomized controlled trial of Lautenschlager et al. (2008) [41]. The standardized mean difference for ADAS-Cog was -1.22 . The drop-out rate was set to $20 \%$. We estimated that a sample size of 46 participants (23 in each group) would provide $95 \%$ power for detecting a significant group difference.

All statistical analyses were conducted per protocol and with the statistical software SPSS version 23 for Windows (IBM, Armonk, NY, United States). Baseline differences between groups regarding anthropometric data, the prevalence of risk factors and physical performance were checked with either independent t-test, 
Mann-Whitney-U-test or $\chi^{2}$-test for categorical variables. Data were z-transformed and statistical outliers (defined as \pm 3 SD from the mean) were excluded for each variable. Normal distribution was checked with the Shapiro-Wilk test $(p>0.05)$, and homoscedasticity with Levene's test $(p>0.05)$. Baseline-adjusted Analyses of Covariance (ANCOVAs) were conducted to determine significant group differences for cognitive and blood biomarkers, with group (golf, control) and time (baseline, post) as the main factors. The specific baseline values as well as age were used as covariates for all analyses. Additionally, the change score of BMI $\left(t_{1}-t_{0}\right)$ was used as a covariate for the analysis of the $\mathrm{KP}$, since visceral fat mass is known to induce systemic low-grade inflammation [23]. Significant time"group interactions were post-hoc analyzed with pairwise Bonferroni-adjusted tests. Due to the explorative character and the small sample size, a larger p-value spectrum was considered for the KP data only [45]. Of note, not all parameters fulfilled the criteria for parametric tests (not normal distributed, heteroscedasticity). ANOVA was shown to be robust against violation of normal distribution, especially when group sizes were over 10 [46]. Transformation of variables to achieve assumptions for parametric tests was also performed, but not associated with different results.

An effect size between Cohen's $d \leq 0.2$ was considered a small effect, Cohen's d 0.2 to 0.5 a medium effect and Cohen's $d>0.8$ a large effect [47]. To explore the association between compliance (overall number of sessions, number of attended third unsupervised training sessions only) and biological markers (IL-6, KP metabolites), Spearman-rank correlations were calculated, with a significance level of $\mathrm{p}<0.05$. The obtained $\mathrm{p}$-values from the correlation analysis were corrected with the False Discovery Rate (FDR) procedure [48]. Due to the explorative character of the study, corrected and uncorrected p-values of the correlation analysis were reported.

\section{Results}

\section{Baseline characteristics}

No statistically significant baseline differences between groups regarding age, gender, education, BDI-score, ADAS-Cog-Score, as well as cardiovascular risk factors and physical outcomes were detected. According to the BDI [40], two participants reported symptoms of mild

Table 1 Demographic information at baseline of analyzed participants $(n=42)$

\begin{tabular}{|c|c|c|c|c|}
\hline & $\begin{array}{l}\text { Golf group } \\
(n=23)\end{array}$ & $\begin{array}{l}\text { Control group } \\
(n=19)\end{array}$ & $p$-value & Cohen's d \\
\hline Age (years) & $67.87 \pm 4.7$ & $67.89 \pm 3.9$ & 0.95 & 0.05 \\
\hline \multicolumn{5}{|l|}{ Gender } \\
\hline male & $10(43.5 \%)$ & $9(47.4 \%)$ & 0.51 & \\
\hline female & $13(56.5 \%)$ & $10(52.6 \%)$ & & \\
\hline \multicolumn{5}{|l|}{ Handedness } \\
\hline right & $22(95.7 \%)$ & $19(100 \%)$ & 0.53 & \\
\hline left & $1(4.3 \%)$ & 0 & & \\
\hline Formal education (years) & $14.4 \pm 4.5$ & $12.26 \pm 3.6$ & 0.13 & 0.59 \\
\hline BDI (score) & $4.7 \pm 4.4$ & $4.4 \pm 3$ & 0.93 & 0.10 \\
\hline ADAS-Cog (score) & $8 \pm 3.55$ & $7.68 \pm 3.41$ & 0.71 & 0.09 \\
\hline \multicolumn{5}{|l|}{ Cardiovascular risk factors } \\
\hline BMI & $26.3 \pm 3.4$ & $26.2 \pm 3.9$ & 0.44 & 0.02 \\
\hline Current smoker & $3(13 \%)$ & $2(10.5 \%)$ & 0.55 & \\
\hline Heavy smoker & $0(0 \%)$ & $1(5.3 \%)$ & 0.48 & \\
\hline Diabetes mellitus type 1 & $2(8.7 \%)$ & $1(5.3 \%)$ & 0.54 & \\
\hline Diabetes mellitus type 2 & $1(4.3 \%)$ & $1(5.3 \%)$ & 0.73 & \\
\hline Hypertension & $12(52.2 \%)$ & $7(36.8 \%)$ & 0.26 & \\
\hline Heart disease & $4(17.4 \%)$ & $3(15.8 \%)$ & 0.55 & \\
\hline \multicolumn{5}{|l|}{ Physical outcomes } \\
\hline 6-min-Walk-Test (m) & $651.37 \pm 121.32$ & $627.73 \pm 99.67$ & 0.48 & 0.24 \\
\hline PASE (score) & $181.11 \pm 59.52$ & $153.37 \pm 57.05$ & 0.15 & 0.48 \\
\hline
\end{tabular}

Quantitative variables were expressed as means \pm standard deviations and categorical variables were expressed as numbers and percentage values Group differences were tested with Mann-Whitney- $U$ for ordinal scaled variables, with independent $t$-test for metric variables, and with $X^{2}$ tests for categorical variables. BDI Becks-Depression-Inventory; ADAS-Cog Alzheimer's Disease Assessment Scale - Cognitive Subscale, BMI Body Mass Index; PASE Physical Activity Scale for Elderly, $p \leq 0.05=$ statistical significance 
depression at baseline (16 points), but were not diagnosed with depression or bipolar disorder. The other participants had no clinically relevant symptoms of depression (<12 points). An overview of baseline characteristics is presented in Table 1.

\section{Subjective memory complaints}

All participants of both groups reported SMC in daily life. $26 \%$ (golf, $n=6$ ) and $32 \%$ (control, $n=6$ ) reported serious worries about the complaints. The onset of memory complaints was 2.6 years ago in the golf group ( \pm 2 years) and also in the control group $( \pm 2.6$ years). After the intervention, $35 \%(n=8)$ of the golf group reported an improvement of these symptoms, while no improvement was reported in the control group. $5.3 \%(n=$ 1) of the control group and no one in the golf group reported a worsening during the 22 weeks. $65 \%(n=15)$ of the golf group and $95 \%(n=18)$ of the control group reported unchanged symptoms.

\section{Primary outcomes: Feasibility and general cognitive performance}

A CONSORT Flow Diagram of the study is presented in Fig. 2. All participants $(n=25)$ of the golf group and 19 participants of the control group took part in the assessments at both time points. Two participants of the control group dropped out without provided reasons. The development of serious diseases during the intervention (brain tumor and motor neuron disease), which were not related to the study, required the exclusion of two participants of the golf group from all analyses. There were no adverse events related to the golf intervention during the study. The overall attendance rate in the golf group was $75 \%$ ( $48 \pm 9.9$ of 65 sessions) and $70 \%$ (15 \pm 5.2 of 22 sessions) for the third training session only. All subjects who undertook the golf exam in the end passed
(20/23) it, but three subjects were unable to attend the test due to limited time. $69 \%$ of all PASE questionnaires were filled out during the observation period in both groups. Self-reported activity levels during the study are presented in Fig. 3.

No significant time or time "group interactions were found for ADAS-Cog $(p=0.613, \mathrm{~F}=0.260$, Cohen's $\mathrm{d}=$ $0.17)$. An overview of the statistical results as well as means and standard deviations at $t_{0}$ and $t_{1}$ is presented in Table 2.

\section{Secondary outcomes: Specific cognitive functions, KP metabolites, and physical performance}

No significant time or time"group interactions were found for TMT B or INHIB reaction time. Significant time effects were found for Corsi forward $(p=0.033, \mathrm{~F}=$ 4.888, Cohen's $\mathrm{d}=0.16)$ and Corsi backwards $(p=0.023$, $\mathrm{F}=5.620$, Cohen's $\mathrm{d}=0$ ). However, after Bonferroniadjusted pairwise post-hoc analysis no significant changes in both groups for these parameters remained. A significant time*group interaction was found for INHIB correct responses $(p=0.012, \mathrm{~F}=7.050$, Cohen's $\mathrm{d}=0.89$ ), while reaction time remained stable in both groups. Bonferroni post-hoc analysis revealed a significant increase in INHIB correct responses in the golf group compared to the control group ( $p=0.012,95 \% \mathrm{CI}$ $0.653,4.834$ ).

No significant time or time* group interactions were found for KYN, TRP, KYNA, QUINA, KYNA/KYN, QUINA/KYNA or QUINA/KYN ratio, IL-6 and for the 6-Min-Walk-Test, but for QUINA/TRP $(p=0.022, \mathrm{~F}=$ 5.769 , Cohen's $\mathrm{d}=0.84)$ in favor of the golf group $(p=$ 0.022, 95 \% CI 0, 0.001).

A trend towards a significant time*group interaction was found for KYN/TRP ratios $(p=0.087, \mathrm{~F}=3.108$, Cohen's $d=0.61)$. Another almost significant

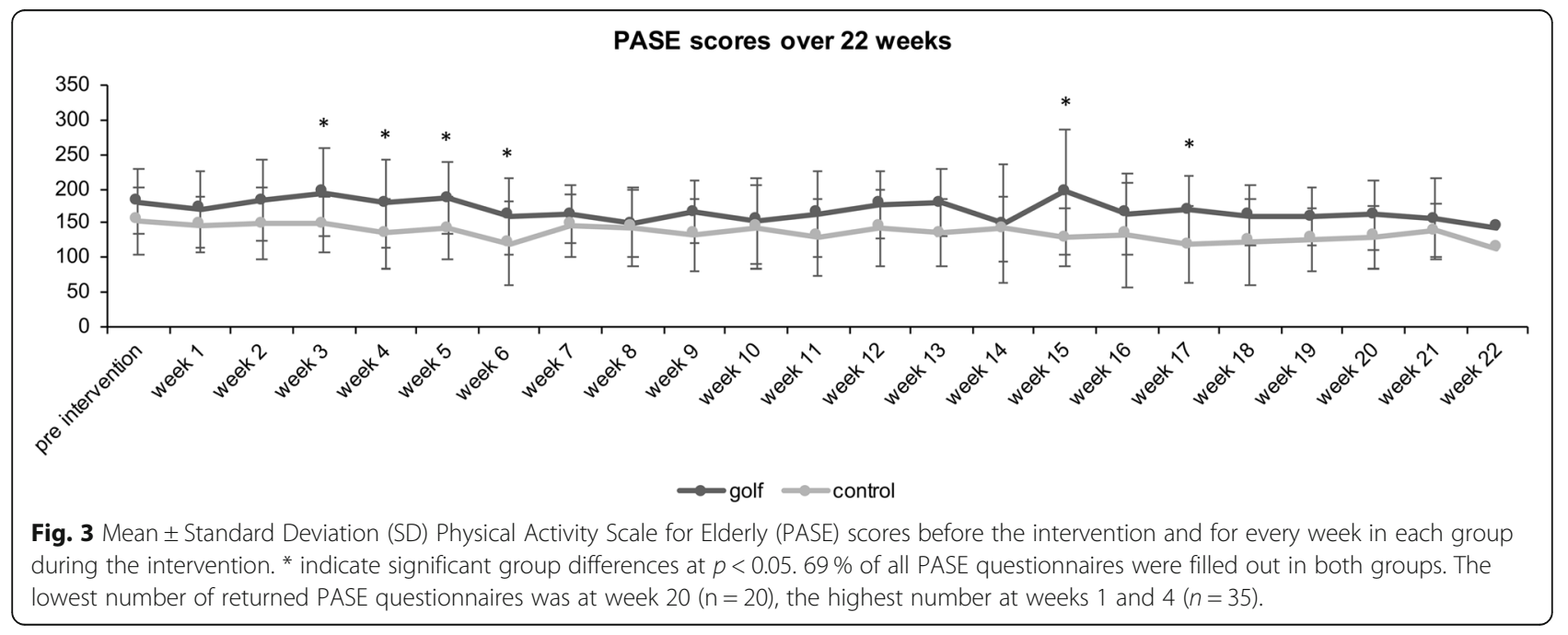


Table 2 Descriptive data indicated as mean and standard deviation (SD) at baseline $\left(t_{0}\right)$ and after the intervention $\left(t_{1}\right)$

\begin{tabular}{|c|c|c|c|c|c|c|}
\hline outcome & group & $\mathrm{n}$ & $t_{0}$ (mean, SD) & $t_{1}($ mean, SD) & ANCOVA time*group ( $p, F$, Cohen's d) & $\begin{array}{l}\text { post-hoc analysis } \\
(p, 95 \% \mathrm{Cl})\end{array}$ \\
\hline \multirow[t]{2}{*}{ ADAS-Cog ${ }^{1}$ (score) } & golf & 22 & $7.45(2.46)$ & $6.82(2.67)$ & $0.613,0.260,0.17$ & \\
\hline & control & 19 & $7.68(3.40)$ & $6.58(2.97)$ & & \\
\hline \multirow[t]{2}{*}{ Corsi forward ${ }^{1}$ (no. of sequences) } & golf & 22 & $7.91(1.31)$ & $7.77(2.02)$ & $0.641,0.221,0.16$ & \\
\hline & control & 19 & $6.74(2.13)$ & $7.16(2.43)$ & & \\
\hline \multirow[t]{2}{*}{ Corsi backwards ${ }^{1}$ (no. of sequences) } & golf & 23 & $6.48(2.35)$ & $6.65(2.77)$ & $0.921,0.010,0$ & \\
\hline & control & 19 & $6.26(2.96)$ & $6.42(3.15)$ & & \\
\hline \multirow[t]{2}{*}{ TMT B time ${ }^{1}(\mathrm{~s})$} & golf & 22 & $49.44(18.87)$ & $47.58(12.57)$ & $0.305,1.085,0.35$ & \\
\hline & control & 18 & $49.81(22.25)$ & $52.66(29.03)$ & & \\
\hline \multirow[t]{2}{*}{ INHIB reaction time ${ }^{1}$ (ms) } & golf & 22 & $0.37(0.05)$ & $0.36(0.05)$ & $0.975,0.000,0$ & \\
\hline & control & 19 & $0.35(0.04)$ & $0.35(0.05)$ & & \\
\hline \multirow[t]{2}{*}{ INHIB correct responses ${ }^{1}(\%)$} & golf & 22 & $91.36(6.84)$ & $95.27(3.24)$ & $\mathbf{0 . 0 1 2} *, 7.050,0.89$ & $\mathbf{0 . 0 0 0 *},(2.075,4.884)$ \\
\hline & control & 18 & $92.87(4.27)$ & $93.07(4.41)$ & & $0.349,(-0.827,2.280)$ \\
\hline \multirow[t]{2}{*}{$\mathrm{KYN}^{2}(\mu \mathrm{mol} / \mathrm{L})$} & golf & 22 & $1.69(0.55)$ & $1.84(0.74)$ & $0.418,0.671,0.28$ & \\
\hline & control & 17 & $2.04(0.99)$ & $2.19(0.85)$ & & \\
\hline \multirow[t]{2}{*}{$\operatorname{TRP}^{2}(\mu \mathrm{mol} / \mathrm{L})$} & golf & 22 & $127.48(25.68)$ & 129.67 (16.39) & $0.464,0.548,0.26$ & \\
\hline & control & 17 & $129.67(20.90)$ & $126.56(21.13)$ & & \\
\hline \multirow[t]{2}{*}{ KYN/TRP ratio ${ }^{2}$} & golf & 22 & $0.014(0.005)$ & $0.014(0.005)$ & $0.087,3.108,0.61$ & \\
\hline & control & 17 & $0.016(0.006)$ & $0.017(0.006)$ & & \\
\hline \multirow[t]{2}{*}{$\mathrm{KYNA}(\mu \mathrm{mol} / \mathrm{L})^{2}$} & golf & 22 & $0.093(0.023)$ & $0.095(0.031)$ & $0.926,0.009,0$ & \\
\hline & control & 17 & $0.105(0.048)$ & $0.105(0.039)$ & & \\
\hline \multirow[t]{2}{*}{ KYNA/KYN ratio ${ }^{2}$} & golf & 22 & $0.058(0.02)$ & $0.057(0.02)$ & $0.598,0.284,0.18$ & \\
\hline & control & 17 & $0.057(0.02)$ & $0.054(0.03)$ & & \\
\hline \multirow[t]{2}{*}{ QUINA $(\mu \mathrm{mol} / \mathrm{L})^{2}$} & golf & 21 & $0.72(0.24)$ & $0.67(0.22)$ & $0.224,1.537,0.43$ & \\
\hline & control & 17 & $0.71(0.24)$ & $0.73(0.19)$ & & \\
\hline \multirow[t]{2}{*}{ QUINA/KYNA ratio ${ }^{2}$} & golf & 21 & $8.20(2.32)$ & $7.38(1.69)$ & $0.227,1.513,0.43$ & \\
\hline & control & 17 & $7.43(2.65)$ & $7.64(2.92)$ & & \\
\hline \multirow[t]{2}{*}{ QUINA/TRP } & golf & 21 & $0.0058(0.002)$ & $0.0051(0.001)$ & $\mathbf{0 . 0 2 2} *, 5.769,0.84$ & $\mathbf{0 . 0 2 2} *(0,0.001)$ \\
\hline & control & 17 & $0.0057(0.002)$ & $0.0059(0.002)$ & & $0.527,(-0.001,0)$ \\
\hline \multirow[t]{2}{*}{ QUINA/KYN } & golf & 21 & $0.456(0.145)$ & $0.406(0.154)$ & $0.869,0.028,0.06$ & \\
\hline & control & 17 & $0.394(0.141)$ & $0.364(0.107$ & & \\
\hline \multirow[t]{2}{*}{$\mathrm{IL}-6(\mathrm{pg} / \mathrm{ml})^{2}$} & golf & 22 & $1.54(0.59)$ & $1.82(0.97)$ & $0.607,0.270,0.18$ & \\
\hline & control & 15 & $2.05(1.17)$ & $2.16(1.33)$ & & \\
\hline \multirow[t]{2}{*}{ 6-Min-Walk-Test ${ }^{1}(\mathrm{~m})$} & golf & 23 & 651.65 (115.75) & $653.58(153.82)$ & $0.439,0.613,0.26$ & \\
\hline & control & 16 & $646.21(91.12)$ & 673.78 (106.33) & & \\
\hline \multirow[t]{2}{*}{ PASE (score) $)^{1}$} & golf & 21 & $180.86(59.02)$ & $171.05(45.72)$ & $0.056,3.888,0.66$ & \\
\hline & control & 19 & 153.37 (57.05) & $137.50(37.91)$ & & \\
\hline
\end{tabular}

Results of baseline-adjusted ANCOVA are presented (time, time*group) with Bonferroni-corrected pairwise post-hoc analysis for each parameter. ${ }^{*}$ indicate statistical significant changes or differences at $p<0.05$. ADAS-Cog Alzheimer's Disease Assessment Scale - Cognitive Subscale, TMT Trail Making Test, INHIB Response Inhibition, KYN Kynurenine, TRP Tryptophan, KYNA Kynurenic acid, QUINA Quinolinic acid, IL-6 Interleukin 6, PASE Physical Activity Scale for Elderly, FDR False Discovery Rate (Benjamini Hochberg Procedure) ' ${ }^{1}$ adjusted for age, ${ }^{2}$ adjusted for age and mean change of BMI $\left(\mathrm{t}_{1}-\mathrm{t}_{0}\right)$

group*time interaction was found for PASE $(p=0.056$, $\mathrm{F}=3.888$, Cohen's $\mathrm{d}=0.66)$. Significant interaction effects as well as trends within the KP are visualized in Fig. 4.
Exploratory Spearman correlation analysis showed a significant negative correlation of delta QUINA/KYNA ratio $\left(\mathrm{t}_{1}-\mathrm{t}_{0}\right)$ with adherence to the third training session in the golf group $\left(p=0.039, \mathrm{r}_{\mathrm{s}}=-0.443\right)$. Of note, the 


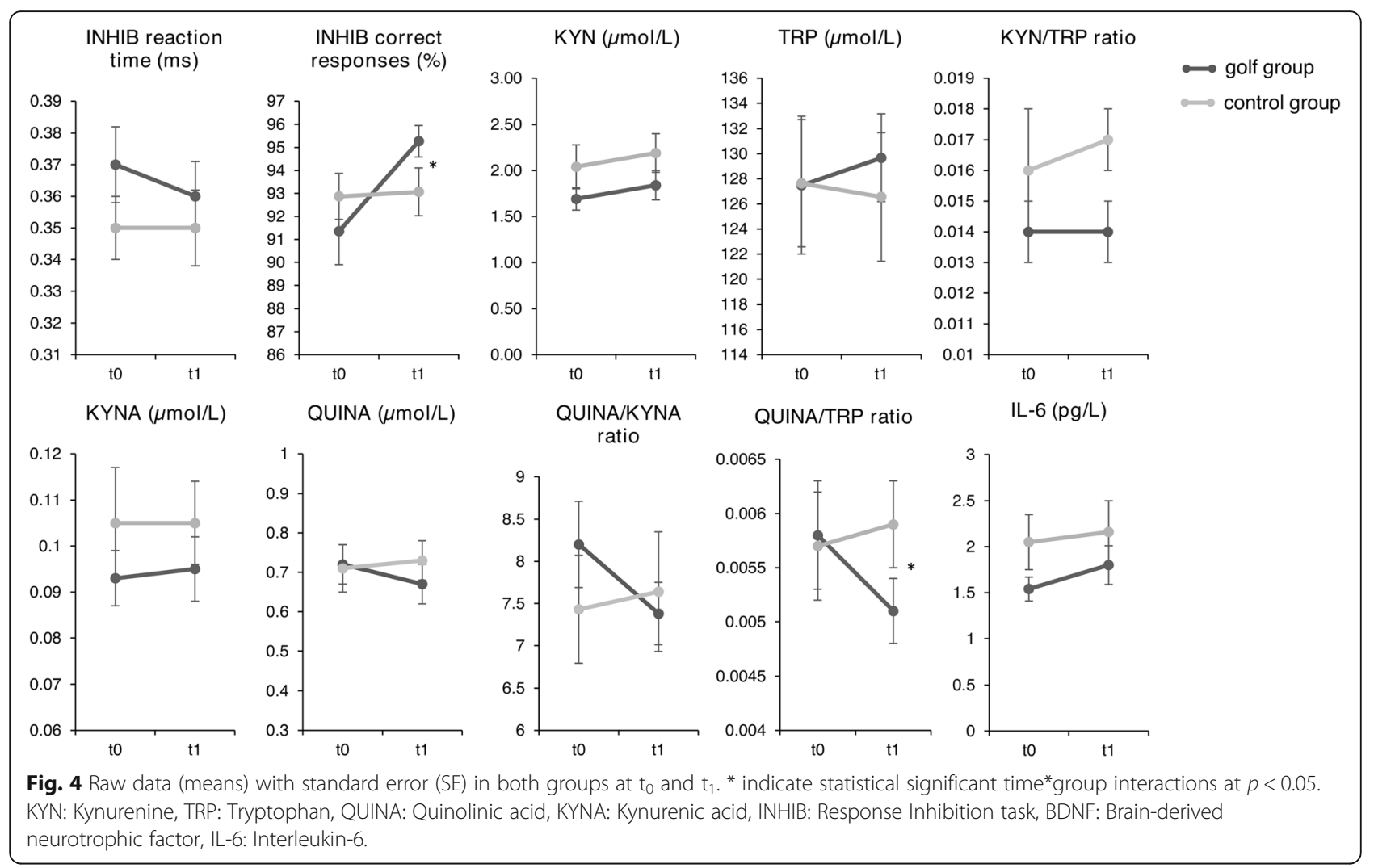

correlation was not significant after FDR correction. An association between the achieved points of the golf exam and the KP was not found. Results of the correlation analyses are presented in Fig. 5.

\section{Discussion}

The aim of this study was to elucidate the feasibility of a 22-weeks golf intervention on the driving range and its effects on cognitive functions and the KP in elderly people with SMC without prior golf experience. We could show that elderly people with cognitive problems were able to learn golf, although it is a complex and difficult sport to learn. No adverse events related to the golf intervention were reported, and no participant in the golf group dropped out. Overall adherence to the golf training was $75 \%$, which can be considered as acceptable. Two participants of the control group dropped out, as they made their participation contingent on allocation into the golf group. Of note, this might have
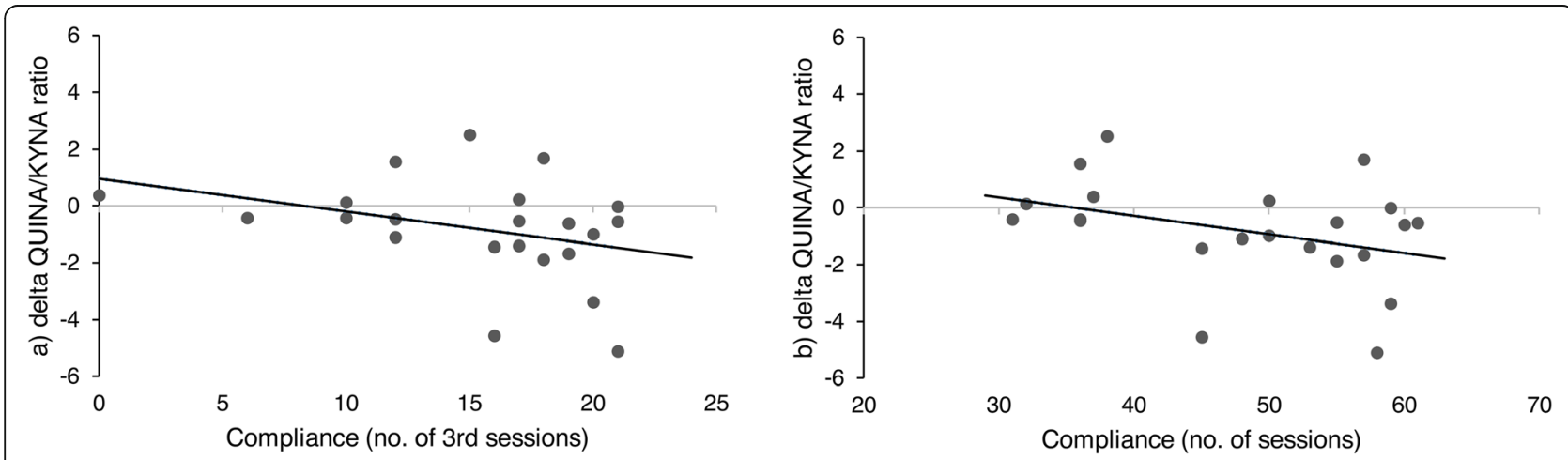

Fig. 5 Results of Spearman correlation analyses. $\mathbf{a}+\mathbf{b}$ : Association of compliance (number of attended sessions) with delta Quinolinic acid to kynurenic acid ratios (QUINA/KYNA ratios, t1 - t0). a: only number of third sessions attended) and delta QUINA/KYNA ratios, $\mathbf{b}$ number of all attended sessions and delta QUINA/KYNA ratios. $\mathbf{a} r_{5}=-0.443, p=0.039, \mathbf{b} r_{5}=-0.400, p=0.065$. Of note, the correlation between compliance (no. of 3rd sessions) and delta QUINA/KYNA ratios was not significant after correcting for type 1 errors $(p=0.039>0.005$ 
influenced comparability of both groups, e.g. regarding physical activity levels (PASE). Three participants were not able to attend the golf exam due to organizational reasons. All other participants passed it (20/23). Therefore, for people with cognitive problems, findings support the idea that learning to play golf is feasible and safe.

Results of this pilot study revealed no significant effects of learning to play golf on general cognitive performance, working memory or other measures of executive functions compared to the control group. Only the number of correct responses on the INHIB task increased significantly in the golf group compared to the control group, while the reaction time remained unchanged. For the INHIB task, participants should only react when a certain stimulus is presented (Go/NoGo). Golfers therefore made less omission (missing reaction) and commission errors (wrong reaction) compared to the control group throughout the task. These findings suggest a small effect on sustained attention.

Shimada et al. (2018) [34] found an improvement in logical memory after 24 weeks golf intervention, which was not an outcome in this study. Learning to play golf requires high demands on motor and cognitive abilities [35] and less on cardiovascular abilities (compared to endurance training). In addition, the golf training consisted mostly of whole-body-coordination exercises at the driving range, which is also indicated by an unchanged endurance performance in the golf group. The findings are in accordance with Voelcker-Rehage et al. (2011) [49], who found a higher accuracy during a Flanker and a Visual Search task in a coordination exercise group. Thus, our results might not reflect the multidimensionality of playing golf, which also includes cardiovascular demands that is induced by walking over the fairway [33]. Evidence from studies investigating the effects of dancing (which is possibly comparable to golf because of its multidimensionality) revealed increases in attention only at 6 months of dance training, whereas episodic memory performance increased after 18 months of training [30]. Improvements in cognitive functions were reportedly not linear [30], and the positive effects of a multidimensional activity on cognitive functions might not be present at 22 weeks of practice. In this study, only data from the 22-week observation is available, which impedes the long-term investigation of all demands of golf on cognitive functions.

On a biological level, learning to play golf showed a positive, but non-significant influence on peripheral KP regulation, which could be of interest for future studies. A significant group*time interaction was only found for QUINA/TRP ratios in favor of the golf group. Descriptive data indicated that KYN levels as well as KYN/TRP ratios remained stable during the intervention in the golf group, but increased almost significant in the control group, suggesting enhanced IDO1 activity. Of note, KYN/TRP ratios could also increase by cortisol due to higher stress levels in the control group, which in turn influences the expression of TDO $[23,50]$. Interestingly, the KYN/TRP ratios observed in this study are lower compared to findings from cross-sectional studies, e.g. in elderly people (median KYN/TRP ratio 0.025, interquartile range 0.006, [20]) and in AD patients (median KYN/ TRP ratio 0.034, interquartile range 0.008, [17]). Besides substantially increased KYN/TRP ratios that have been described in neurodegenerative diseases [51], the participants in Solvang et al. [20] were on average 10 years older compared to this sample, which might be one explanation for the higher values. To the best of our knowledge, no cut-off value exists yet that indicates an increased risk for future cognitive decline or $\mathrm{AD}$.

The non-significant increase of KYN/TRP ratios could also be the result of decreased habitual physical activity in the control group during the observation period, as indicated by PASE scores (see Fig. 2). The activity associated with regular golf training therefore might have the potential to counteract age or AD-related changes of IDO1 activity. This is supported by the non-significant decrease of serum QUINA levels as well as serum QUINA/KYNA ratios golf group only, which might indicate a reduction of Kynurenine 3-monooxygenase (KMO) activity [50, 52]. IDO1 and KMO activity are both driven by inflammatory states $[13,53]$. Of note, the findings did not reach statistical significance, and should therefore be replicated in a larger sample. In addition, it cannot be ruled out that the results are affected by other enzymes of the KP, such as Kynureninase or Kynurenine aminotransferase (KAT).

We also found a negative association between delta QUINA/KYNA ratios and the number of golf training sessions, indicating a positive influence of training frequency on the KP. Of note, the correlation was not significant after Benjamini Hochberg-correction of type 1 error and should also be interpreted carefully.

One randomized controlled trial exists which investigated the effects of a multicomponent exercise intervention (endurance, coordination, balance, flexibility, strength) in elderly at risk of dementia. Küster et al. [21] did not find effects of a 10-week multicomponent exercise intervention on KP metabolites either. Similar to our intervention, the metabolic demands might have been too low to trigger mechanisms that were described regarding the positive effects of exercise on the KP [23], as KYN remained unchanged in the golf group. The positive effects of learning to play golf on the KP might be rather mediated by reduced systemic inflammation, stress or other mechanisms, which are reported effects of physical activity $[25,54]$. 
Our study has some limitations, which should be considered when interpreting the results. First, the sensitivity of the ADAS-Cog might have been too low to detect cognitive changes in this cohort. However, this test was chosen as the primary outcome to allow comparability to previous studies with similar cohorts (e.g. [55-57]). Second, the sample was active (according to the PASE questionnaire, $[58,59]$ ), physically fit (according to the 6MWT, [42]) and well educated $(13.5 \pm 4.2$ years on average), which indicates a selection bias that may affect the generalizability of the results. Of note, it would be of great interest to motivate sedentary elderly people, which are not already physically active, to take part in such a program. In recent years, accessibility of golf was increased by modifying rules of the game, the golf course as well as the equipment for people with various impairments [60]. Moreover, playing golf can facilitate establishment of social relationships, which in turn increases motivation to continue with the sports.

Third, self-reported and cognitive outcomes in this study might be prone to reflect placebo effects or other factors related to the intervention, such as an increased social interaction, because blinding of the participants was not possible and there was no adequate treatment in the control group. The results should therefore be interpreted carefully.

Accordingly, we did not achieve the calculated sample size because many of the screened subjects made their participation in the study contingent on a randomization into the golf group and therefore had to be excluded. The study is therefore underpowered, which might have led to the absence of the effects of golf on neuropsychological outcomes and the KP. Thus, our findings should be replicated in a larger, more representative sample and rather be interpreted as preliminary results. Subgroup analyses of the effects of golf on cognitive functions and the KP accounting for particular concerns and gender differences would have also been useful [4]. In this context, it was recently shown that elderly women with high cortical amyloid- $\beta$ burden also had higher serum KYN levels and KYN/TRP ratios [11]. Serious concerns about SMC were further associated with quantitative amyloid$\beta$ deposition [10] and it was suggested, that the decline in estrogen levels after menopause might increase the vulnerability to KP activity and AD [11]. In our study IL-6 was used as a surrogate marker for inflammatory processes that may influence KP regulation via upregulation of IDO or TDO. However, further studies should include a broader variability of markers of neurodegeneration, neuroinflammation and neuroplasticity.

People with SMC were diagnosed according to guidelines published in [39]. There is some evidence that selfreported SMC can predict future cognitive decline [61], but findings are controversial [62]. Thus, it remains unclear if the study population is relevant for AD or if SMC had other etiologies.

Participants were not scheduled at the exact same time of the day at baseline and after the intervention for the blood sample collection and chronobiological effects might not be fully excluded. However, moderate to strong correlations of $t_{0}$ and $t_{1}$ IL- 6 and KP parameters do not support relevant effects. Participants were not fasted during blood sample collection, which might have influenced the outcome measures as well. Therefore, future studies should conduct measurements with standardized conditions.

In general, causality of central changes of the KP by the golf intervention cannot be assumed, but has to be elucidated in further studies [21].

Since the aim of this study was to assess effects of a complex multicomponent activity in analogy to the enriched environment animal model, we are limited in concluding which stimulation (cognitive, social, sensory, motor) had the largest effects on cognitive outcomes and the KP or whether the combination of all the above is most effective. However, the current literature supports positive effects of recreational and physical activities in natural green environments on mental health in elderly people [63] and attention performance [64].

\section{Conclusions}

The findings of this randomized trial support the idea that learning the challenging sports golf with a multidimensional profile is feasible and safe for people with SMC. Preliminary results revealed improved attention performance and a trend towards a positive influence on the regulation of the KP in the golf group. To elucidate the effects of long-term golf training and the potential to reduce cognitive decline and associated changes in the $\mathrm{KP}$, studies should replicate the findings in a larger and less active sample of elderly people with SMC over a longer period, including higher metabolic demands by playing on the golf course.

\footnotetext{
Abbreviations

AD: Alzheimer's Disease; ADAS-Cog: Alzheimer's Disease Assessment Scale Cognitive Subscale; ANCOVA: Analysis of Covariance; ANOVA: Analysis of Variance; BDI: Beck's Depression Inventory; BMI: Body-Mass-Index; CNS: Central nervous system; CONSORT: Consolidated Standards of Reporting Trials; ELISA: Enzyme-linked Immunosorbent Assay; FDR: False Discovery Rate; HPLC: High performance liquid chromatography; IDO1: Indoleamine-2,3Dioxygenase 1; IL-6: Interleukin-6; INHIB: Response Inhibition Task; KAT: Kynurenine Amino Transferase; KMO: Kynurenine 3-monooxygenase; KP: Kynurenine Pathway; KYN: Kynurenine; KYNA: Kynurenic Acid; $\mathrm{NAD+}$ : Nicotinamide adenine dinucleotide (oxidized form); PASE: Physical Activity Scale for Elderly; PGC-1a: Peroxisome proliferator-activated receptor gamma coactivator 1-alpha; PGA: Professional Golfers Association; QUINA: Quinolinic Acid; SD: Standard Deviation; SMC: Subjective Memory Complaints; TDO: Tryptophan 2,3-Dioxygenase; TMT: Trail Making Test; TRP: Tryptophan; 6MWT: 6-Minute Walk Test; 3-HK: 3-hydroxykynurenine; 3HAA: 3-hydroxyanthranilic acid
} 


\section{Acknowledgements}

We are thankful for the support of Caren Strote and Jessica Michels during data collection.

\section{Authors' contributions}

JKS, SV, FvdB and CR contributed to the study conception and design. JKS, FvdB and CG performed data collection. AS and PZ processed the blood data. Data analysis was conducted by JKS with support from AS and PZ. MO reviewed the statistical methods and analyses. JKS and CR wrote the manuscript and all authors commented on previous versions. All authors read and approved the final manuscript.

\section{Funding}

The golf training was financially supported by the Heinz Nixdorf Westfalian foundation. Open Access funding enabled and organized by Projekt DEAL.

\section{Availability of data and materials}

The datasets used and/or analysed during the current study are available from the corresponding author on reasonable request.

\section{Declarations}

\section{Ethics approval and consent to participate}

The protocol was approved by the ethics committee of the "Westfälische Wilhelms-Universität Münster". Written informed consent to participate in the study was obtained by each participant before enrollment and was in accordance with the Declaration of Helsinki.

\section{Consent for publication}

Not applicable.

\section{Competing interests}

The golf training was financially supported by the Heinz Nixdorf Westfalian foundation.

The authors declare no competing interests.

\section{Author details}

${ }^{1}$ Department of Sports and Health, Institute of Sports Medicine, Paderborn University, Warburger Straße 100, 33098 Paderborn, Germany. ${ }^{2}$ Department for Performance and Health, Institute for Sport and Sport Science, Technical University Dortmund, Otto-Hahn-Straße 3, 44227 Dortmund, Germany. ${ }^{3}$ Institute of Medical Statistics and Computational Biology, Medical Faculty and University Hospital of Cologne, University of Cologne,

Robert-Koch-Straße 10, 50931 Cologne, Germany.

\section{Received: 26 November 2020 Accepted: 24 March 2021}

Published online: 17 May 2021

\section{References}

1. Mitchell AJ. The clinical significance of subjective memory complaints in the diagnosis of mild cognitive impairment and dementia: a meta-analysis. Int J Geriatr Psychiatry. 2008;23:1191-202. doi:https://doi.org/10.1002/gps.2053.

2. Mitchell AJ, Beaumont H, Ferguson D, Yadegarfar M, Stubbs B. Risk of dementia and mild cognitive impairment in older people with subjective memory complaints: meta-analysis. Acta Psychiatrica Scandinavica. 2014;130: 439-51. doi:https://doi.org/10.1111/acps.12336.

3. Bhome R, Berry AJ, Huntley JD, Howard RJ. Interventions for subjective cognitive decline: systematic review and meta-analysis. BMJ Open. 2018;8: e021610. doi:https://doi.org/10.1136/bmjopen-2018-021610.

4. Weber MT, Mapstone M, Staskiewicz J, Maki PM. Reconciling subjective memory complaints with objective memory performance in the menopausal transition. Menopause. 2012;19:735-41. doi:https://doi.org/10.1 097/gme.0b013e318241fd22.

5. Yates JA, Clare L, Woods RT. Subjective memory complaints, mood and MCl: a follow-up study. Aging Ment Health. 2017;21:313-21. doi:https://doi.org/1 0.1080/13607863.2015.1081150.

6. Paradise MB, Glozier NS, Naismith SL, Davenport TA, Hickie IB. Subjective memory complaints, vascular risk factors and psychological distress in the middle-aged: a cross-sectional study. BMC Psychiatry. 2011;11:108. doi: https://doi.org/10.1186/1471-244X-11-108.
7. Yap KH, Warren N, Allotey P, Reidpath DD. Chronic disease profiles of subjective memory complaints: a latent class analysis of older people in a rural Malaysian community. Aging Ment Health. 2020;24:709-16. doi:https:// doi.org/10.1080/13607863.2018.1550632

8. Snitz BE, Lopez OL, McDade E, Becker JT, Cohen AD, Price JC, et al. Amyloid$\beta$ Imaging in Older Adults Presenting to a Memory Clinic with Subjective Cognitive Decline: A Pilot Study. J Alzheimers Dis. 2015;48 Suppl 1:S151-9. doi:https://doi.org/10.3233/JAD-150113.

9. Rönnlund M, Sundström A, Adolfsson R, Nilsson L-G. Subjective memory impairment in older adults predicts future dementia independent of baseline memory performance: Evidence from the Betula prospective cohort study. Alzheimers Dement. 2015;11:1385-92. doi:https://doi.org/10.1 016/j.jalz.2014.11.006.

10. Verfaillie SCJ, Timmers T, Slot RER, van der Weijden CWJ, Wesselman LMP, Prins $N D$, et al. Amyloid- $\beta$ Load Is Related to Worries, but Not to Severity of Cognitive Complaints in Individuals With Subjective Cognitive Decline: The SCIENCe Project. Front Aging Neurosci. 2019;11:7. doi:https://doi.org/10.3389/fnagi.2019.00007.

11. Chatterjee P, Goozee K, Lim CK, James I, Shen K, Jacobs KR, et al. Alterations in serum kynurenine pathway metabolites in individuals with high neocortical amyloid- $\beta$ load: A pilot study. Sci Rep. 2018;8:8008. doi:https:// doi.org/10.1038/s41598-018-25968-7.

12. Schultz SA, Oh JM, Koscik RL, Dowling NM, Gallagher CL, Carlsson CM, et al. Subjective memory complaints, cortical thinning, and cognitive dysfunction in middle-aged adults at risk for AD. Alzheimers Dement (Amst). 2015;1:3340. doi:https://doi.org/10.1016/j.dadm.2014.11.010.

13. Chen Y GGJ. Kynurenine Pathway Metabolites in Humans: Disease and Healthy States. International Journal of Tryptophan Research. 2009:1-19.

14. Greilberger J, Fuchs D, Leblhuber F et al. Carbonyl Proteins as a Clinical Marker in Alzheimer's Disease and its Relation to Tryptophan Degradation and Immune Activation. Clin Lab. 2010;56:441-8.

15. Cervenka I, Agudelo LZ, Ruas JL. Kynurenines: Tryptophan's metabolites in exercise, inflammation, and mental health. Science 2017. doi:https://doi. org/10.1126/science.aaf9794.

16. Schwarcz R, Stone TW. The kynurenine pathway and the brain: Challenges, controversies and promises. Neuropharmacology. 2017;112:237-47. doi: https://doi.org/10.1016/j.neuropharm.2016.08.003.

17. Giil LM, Midttun $\varnothing$, Refsum $H$, Ulvik A, Advani R, Smith AD, Ueland PM. Kynurenine Pathway Metabolites in Alzheimer's Disease. J Alzheimers Dis. 2017;60:495-504. doi:https://doi.org/10.3233/JAD-170485.

18. Schwarz MJ, Guillemin GJ, Teipel SJ, Buerger K, Hampel H. Increased 3hydroxykynurenine serum concentrations differentiate Alzheimer's disease patients from controls. Eur Arch Psychiatry Clin Neurosci. 2013;263:345-52. doi:https://doi.org/10.1007/s00406-012-0384-X.

19. Hartai Z, Juhász A, Rimanóczy A, Janáky T, Donkó T, Dux L, et al. Decreased serum and red blood cell kynurenic acid levels in Alzheimer's disease. Neurochem Int. 2007;50:308-13. doi:https://doi.org/10.1016/j.neuint.2006.08.012.

20. Solvang S-EH, Nordrehaug JE, Tell GS, Nygård O, McCann A, Ueland PM, et al. The kynurenine pathway and cognitive performance in communitydwelling older adults. The Hordaland Health Study. Brain Behav Immun. 2019;75:155-62. doi:https://doi.org/10.1016/j.bbi.2018.10.003.

21. Küster OC, Laptinskaya D, Fissler P, Schnack C, Zügel M, Nold V, et al. Novel Blood-Based Biomarkers of Cognition, Stress, and Physical or Cognitive Training in Older Adults at Risk of Dementia: Preliminary Evidence for a Role of BDNF, Irisin, and the Kynurenine Pathway. J Alzheimers Dis. 2017;59: 1097-111. doi:https://doi.org/10.3233/JAD-170447.

22. Hörder H, Johansson L, Guo X, Grimby G, Kern S, Östling S, Skoog I. Midlife cardiovascular fitness and dementia: A 44-year longitudinal population study in women. Neurology. 2018;90:e1298-e1305. doi:https://doi.org/1 0.1212/WNL.0000000000005290.

23. Metcalfe AJ, Koliamitra C, Javelle F, Bloch W, Zimmer P. Acute and chronic effects of exercise on the kynurenine pathway in humans - A brief review and future perspectives. Physiol Behav. 2018;194:583-7. doi:https://doi.org/1 0.1016/j.physbeh.2018.07.015

24. Erickson KI, Voss MW, Prakash RS, Basak C, Szabo A, Chaddock L, et al. Exercise training increases size of hippocampus and improves memory. Proc Natl Acad Sci U S A. 2011;108:3017-22. doi:https://doi.org/10.1073/pnas.1015950108.

25. Mee-Inta O, Zhao Z-W, Kuo Y-M. Physical Exercise Inhibits Inflammation and Microglial Activation. Cells 2019. doi:https://doi.org/10.3390/cells8070691.

26. Dantzer R. Role of the Kynurenine Metabolism Pathway in InflammationInduced Depression: Preclinical Approaches. Curr Top Behav Neurosci. 2017; 31:117-38. doi:https://doi.org/10.1007/7854_2016_6. 
27. van Praag Henriette, Kempermann Gerd, Gage Fred H. Neural Consequences of Environmental Enrichment. Nat Rev Neurosci. 2000;1:1918.

28. Kempermann G, Fabel K, Ehninger D, Babu H, Leal-Galicia P, Garthe A, Wolf SA. Why and how physical activity promotes experience-induced brain plasticity. Front Neurosci. 2010;4:189. doi:https://doi.org/10.3389/fnins.2010. 00189.

29. Sáez de Asteasu ML, Martínez-Velilla N, Zambom-Ferraresi F, Casas-Herrero Á, Izquierdo M. Role of physical exercise on cognitive function in healthy older adults: A systematic review of randomized clinical trials. Ageing Res Rev. 2017;37:117-34. doi:https://doi.org/10.1016/j.arr.2017.05.007.

30. Müller P, Rehfeld K, Schmicker M, Hökelmann A, Dordevic M, Lessmann V, et al. Evolution of Neuroplasticity in Response to Physical Activity in Old Age: The Case for Dancing. Front Aging Neurosci. 2017:9:56. doi:https://doi. org/10.3389/fnagi.2017.00056

31. Rehfeld K, Lüders A, Hökelmann A, Lessmann V, Kaufmann J, Brigadski T, et al. Dance training is superior to repetitive physical exercise in inducing brain plasticity in the elderly. PLOS ONE. 2018;13:e0196636. doi:https://doi. org/10.1371/journal.pone.0196636.

32. Ströhlein JK, van den Bongard F, Barthel T, Reinsberger C. Dose-responserelationship between physical activity and cognition in elderly. Dtsch Z Sportmed. 2017;2017:234-42. doi:https://doi.org/10.5960/dzsm.2017.300.

33. Murray AD, Daines L, Archibald D, Hawkes RA, Schiphorst C, Kelly $P$, et al. The relationships between golf and health: a scoping review. $\mathrm{Br} J$ Sports Med. 2017;51:12-9. doi:https://doi.org/10.1136/bjsports-2016-096625.

34. Shimada H, Lee S, Akishita M, Kozaki K, lijima K, Nagai K, et al. Effects of golf training on cognition in older adults: a randomised controlled trial. J Epidemiol Community Health. 2018;72:944-50. doi:https://doi.org/10.1136/ jech-2017-210052.

35. Jäncke L, Koeneke S, Hoppe A et al. The Architecture of the Golfer's Brain. PLOS ONE. 2009;4:e4785. doi:https://doi.org/10.1371/journal.pone.0004785. t001.

36. Schachten $T$, Jansen $P$. The effects of golf training in patients with stroke: a pilot study. Int Psychogeriatr. 2015;27:865-73. doi:https://doi.org/10.1017/S1 041610214002452.

37. Eldridge SM, Chan CL, Campbell MJ, Bond CM, Hopewell S, Thabane L, Lancaster GA. CONSORT 2010 statement: extension to randomised pilot and feasibility trials. BMJ. 2016;355:i5239. doi:https://doi.org/10.1136/bmj.i5239.

38. Rosen WG, Mohs RC, Davis KL. A new rating scale for Alzheimer's disease. Am J Psychiatry. 1984;141:1356-64. doi:https://doi.org/10.1176/ajp.141.11.1356.

39. Molinuevo JL, Rabin LA, Amariglio R, Buckley R, Dubois B, Ellis KA, et al. Implementation of subjective cognitive decline criteria in research studies. Alzheimers Dement. 2017;13:296-311. doi:https://doi.org/10.1016/j.jalz.2016. 09.012.

40. Beck AT, Ward CH, Mendelson M, Mock J, Erbaugh J. An inventory for measuring depression. Arch Gen Psychiatry. 1961;4:561-71. doi:https://doi. org/10.1001/archpsyc.1961.01710120031004.

41. Lautenschlager NT, Cox KL, Flicker L, Foster JK, van Bockxmeer FM, Jianguo X, et al. Effect of Physical Activity on Cognitive Function in Older Adults at Risk for Alzheimer Disease: A Randomized Trial. Journal of the American Medical Association: JAMA. 2008;300:1027-37.

42. Enright PL, McBurnie MA, Bittner V, Tracy RP, McNamara R, Arnold A, Newman AB. The 6-min Walk Test*. Chest. 2003;123:387-98. doi:https://doi. org/10.1378/chest.123.2.387.

43. Joisten N, Kummerhoff F, Koliamitra C, Schenk A, Walzik D, Hardt L, Knoop A, Thevis M, Kiesl D, Metcalfe AJ, Bloch W, Zimmer P. Exercise and the Kynurenine pathway: Current state of knowledge and results from a randomized cross-over study comparing acute effects of endurance and resistance training. Exercise Immunology Review. 2020;26.

44. Faul F, Erdfelder E, Lang AG, Bichner. G*Power 3: A flexible statistical power analysis program for the social, behavioral, and biomedical sciences. Behav Res Methods. 2007;39:175-91.

45. Thiese MS, Ronna B, Ott U. P value interpretations and considerations. J Thorac Dis. 2016;8:E928-E931. doi:https://doi.org/10.21037/jtd.2016.08.16.

46. Schmider E, Ziegler M, Danay E, Beyer L, Bühner M. Is It Really Robust? Methodology. 2010;6:147-51. doi:https://doi.org/10.1027/1614-2241/a000016.

47. Lenhard W, Lenhard, A. Computation of Effect Sizes. 2016. Available at: https://www.psychometrica.de/effect_size.html. Accessed 28 Oct 2019.

48. Benjamini Y, Hochberg Y. Controlling the False Discovery Rate: A Practical and Powerful Approach to Multiple Testing. Journal of the Royal Statistical
Society: Series B (Methodological). 1995;57:289-300. doi:https://doi.org/1 0.1111/j.2517-6161.1995.tb02031.x.

49. Voelcker-Rehage C, Godde B, Staudinger UM. Cardiovascular and coordination training differentially improve cognitive performance and neural processing in older adults. Front Hum Neurosci. 2011;5:26. doi:https:// doi.org/10.3389/fnhum.2011.00026.

50. Zimmer P, Schmidt ME, Prentzell MT, Berdel B, Wiskemann J, Kellner KH, et al. Resistance Exercise Reduces Kynurenine Pathway Metabolites in Breast Cancer Patients Undergoing Radiotherapy. Front Oncol. 2019;9:962. doi: https://doi.org/10.3389/fonc.2019.00962.

51. Chen Y, Guillemin GJ. Kynurenine pathway metabolites in humans: disease and healthy States. Int J Tryptophan Res. 2009;2:1-19. doi:https://doi.org/1 0.4137/ijtr.s2097.

52. Herrstedt A, Bay ML, Simonsen C, Sundberg A, Egeland C, Thorsen-Streit S, et al. Exercise-mediated improvement of depression in patients with gastroesophageal junction cancer is linked to kynurenine metabolism. Acta Oncol. 2019;58:579-87. doi:https://doi.org/10.1080/0284186X.2018.1558371.

53. Parrott JM, O'Connor JC. Kynurenine 3-Monooxygenase: An Influential Mediator of Neuropathology. Front Psychiatry. 2015;6:116. doi:https://doi. org/10.3389/fpsyt.2015.00116.

54. Tari AR, Norevik CS, Scrimgeour NR, Kobro-Flatmoen A, Storm-Mathisen J, Bergersen $\mathrm{LH}$, et al. Are the neuroprotective effects of exercise training systemically mediated? Prog Cardiovasc Dis. 2019;62:94-101. doi:https://doi. org/10.1016/j.pcad.2019.02.003.

55. Suzuki T, Shimada H, Makizako H, Doi T, Yoshida D, Ito K, et al. A randomized controlled trial of multicomponent exercise in older adults with mild cognitive impairment. PLoS ONE. 2013;8:e61483. doi:https://doi.org/1 0.1371/journal.pone.0061483.

56. Park H, Park JH, Na HR, Hiroyuki S, Kim GM, Jung MK, et al. Combined Intervention of Physical Activity, Aerobic Exercise, and Cognitive Exercise Intervention to Prevent Cognitive Decline for Patients with Mild Cognitive Impairment: A Randomized Controlled Clinical Study. J Clin Med 2019. doi: https://doi.org/10.3390/jcm8070940.

57. Lautenschlager NT, Cox KL, Flicker L, Foster JK, van Bockxmeer FM, Xiao J, et al. Effect of physical activity on cognitive function in older adults at risk for Alzheimer disease: a randomized trial. JAMA. 2008;300:1027-37. doi: https://doi.org/10.1001/jama.300.9.1027.

58. Logan SL, Gottlieb BH, Maitland SB, Meegan D, Spriet LL. The Physical Activity Scale for the Elderly (PASE) questionnaire; does it predict physical health? Int J Environ Res Public Health. 2013;10:3967-86. doi:https://doi. org/10.3390/ijerph10093967.

59. Washburn RA, Smith KW, Jette AM, Janney CA. The physical activity scale for the elderly (PASE): Development and evaluation. Journal of Clinical Epidemiology. 1993;46:153-62. doi:https://doi.org/10.1016/0895-4356(93)90053-4.

60. Parziale JR. Golf in the United States: an evolution of accessibility. PM R. 2014;6:825-7. doi:https://doi.org/10.1016/j.pmrj.2014.04.002.

61. Vaskivuo L, Hokkanen L, Hänninen T, Antikainen R, Bäckman L, Laatikainen T, et al. Self and Informant Memory Reports in FINGER: Associations with TwoYear Cognitive Change. J Alzheimers Dis. 2019;71:785-95. doi:https://doi. org/10.3233/JAD-190133.

62. Nicholas CR, Dowling NM, Racine AM, Clark LR, Berman SE, Koscik RL, et al. Longitudinal Assessment of Self- and Informant-Subjective Cognitive Complaints in a Sample of Healthy Late-Middle Aged Adults Enriched with a Family History of Alzheimer's Disease. J Int Neuropsychol Soc. 2017;23: 617-26. doi:https://doi.org/10.1017/S1355617717000509.

63. Wu Y-T, Prina AM, Jones A, Matthews FE, Brayne C. Older people, the natural environment and common mental disorders: cross-sectional results from the Cognitive Function and Ageing Study. BMJ Open. 2015;5:e007936. doi:https://doi.org/10.1136/bmjopen-2015-007936.

64. Bowler DE, Buyung-Ali LM, Knight TM, Pullin AS. A systematic review of evidence for the added benefits to health of exposure to natural environments. BMC Public Health. 2010;10:456. doi:https://doi.org/10.11 86/1471-2458-10-456.

\section{Publisher's Note}

Springer Nature remains neutral with regard to jurisdictional claims in published maps and institutional affiliations. 